\title{
Réadaptation, desserrement et rénovation des villages d'openfield en Lorraine
}

Adaptation, Opening-up and Renovation of Openfield Villages in Lorraine Anpassung, Auflockerung und Erneuerung der Openfield-Dörfer in Lothringen

Mathieu Huguenin et Jean-Pierre Husson

\section{(2) OpenEdition}

\section{Journals}

Édition électronique

URL : http://journals.openedition.org/rge/2618

DOI : $10.4000 /$ rge.2618

ISSN : $2108-6478$

Éditeur

Association des géographes de l'Est

Édition imprimée

Date de publication : 1 juin 2002

ISSN : 0035-3213

\section{Référence électronique}

Mathieu Huguenin et Jean-Pierre Husson, « Réadaptation, desserrement et rénovation des villages d'openfield en Lorraine », Revue Géographique de l'Est [En ligne], vol. 42 / 3 | 2002, mis en ligne le 14 décembre 2010, consulté le 08 septembre 2020. URL : http://journals.openedition.org/rge/2618; DOI : https://doi.org/10.4000/rge.2618

Ce document a été généré automatiquement le 8 septembre 2020

Tous droits réservés 


\title{
Réadaptation, desserrement et rénovation des villages d'openfield en Lorraine
}

\author{
Adaptation, Opening-up and Renovation of Openfield Villages in Lorraine \\ Anpassung, Auflockerung und Erneuerung der Openfield-Dörfer in Lothringen
}

Mathieu Huguenin et Jean-Pierre Husson

1 Les villages d'openfield ont été passionnément étudiés par les géographes qui ont dressé des typologies classiques connues surtout sous leur terminologie allemande (Strassendorf, Reihendorf, Ringdorf). A partir des années soixante, un important mouvement s'est dessiné pour étudier la genèse de ces villages sur le territoire lorrain et tenter de faire un état des lieux des bâtis devenus trop vastes et parfois obsolètes par rapport au temps du maximum démographique qui s'était imposé plus d'un siècle auparavant ${ }^{1}$.

2 Le village, " engraissé au XVIII ${ }^{\mathrm{e}}$ siècle » (de Planhol 1969), comblé au XIX ${ }^{\mathrm{e}}$ siècle en respectant la logique de notabilité plaçant les laboureurs au centre, les manouvriers en périphérie, subit à partir de 1960 les outrages du passage forcé à une révolution agricole de grande ampleur que F. Braudel n'hésite pas à qualifier de «Grand chambardement ». DES et maîtrises rédigés à cette époque à Nancy montrent une volonté de connaître l'état des lieux de l'existant, qui n'est pas encore considéré comme du patrimoine. Il est souvent balayé par les impératifs techniques qui s'imposent avec le déclin de la population et l'appauvrissement lié au départ sélectif de la majorité des jeunes.

3 On est là bien loin des formes de renaissance rurale (Kayser, 1990, 1996) qui s'esquissent, puis s'amplifient dès la fin de la période de prospérité.

4 Les images évoquées appartiennent à des passés révolus. Actuellement, les villages renaissent en accueillant, dans des cycles et des dosages qui peuvent être très différents de l'un à l'autre, une population désormais majoritairement non agricole. Pour être en cohérence, en continuité avec les images passées qui ont été évoquées, les analyses 
développées dans cet article porteront sur des espaces restés ruraux, placés au-delà du périmètre de $15 \mathrm{~km}$ qui débute, comme l'impose la loi SRU (loi de solidarité du renouvellement urbain) avec la limite de l'habitat dense des villes pour former une zone transitoire à conforter qu'il est aisé d'appeler archipel urbain.

Le rural est au-delà de cette zone directement en synergie avec la ville. Cette limite légale est établie dans une logique d'aménagement, de cohérence territoriale. Une majorité des résidents localisés en dehors de l'archipel urbain sont également des navetteurs, des ruraux travaillant à la ville ou dans les banlieues logistiques qui regroupent beaucoup d'emplois.

Depuis quelque trois décennies, les villages ont changé. Ils s'inscrivent dans une logique de renaissance qui revêt des aspects multiples mais inégaux en fonction de la pertinence des projets de développement et de maîtrise du foncier qui s'y préparent. Cette renaissance est indissociable des refontes parcellaires réalisées dans le domaine agricole. Trois mouvements parfois simultanés, parfois successifs organisent la mise en scène des villages qui, au total, apparaissent plutôt disparates si l'on compare leur état à celui des périodes anciennes. La communauté villageoise tente d'abord d'améliorer la traversée du bâti pour répondre au passage des véhicules chaque fois qu'un contournement ne peut être réalisé (avec l'application d'un remembrement article 10). Elle aménage des espaces fédératifs pour faire naître des lieux de convivialité largement inspirés de la tradition. En même temps, ou plus souvent encore avec un décalage par rapport aux choix évoqués, le village s'étoffe, grandit de façon parfois anarchique, linéaire, ou sous la forme de petits lotissements pavillonnaires. En dernier lieu, le village qui s'est développé et modifié par la requalification des usoirs s'est aussi partiellement dénaturé en perdant ses fonctions agricoles souvent déplacées en périphérie, voire loin des maisons pour répondre au cahier des charges des exigences sanitaires imposées.

7 Au total, l'espace villageois a beaucoup changé et obéit à des logiques d'intérêts multiples qui se dessinent à diverses échelles, convergent ou s'excluent, et posent des questions sur les choix de cadre de vie à faire évoluer dans un souci de fonctionnalité et de cohérence patrimoniale à préserver. L'article associe les regards croisés du géographe et de l'architecte bâtisseur, mais également metteur en scène des cohérences paysagères pour observer les mutations présentes et à venir des villages.

\section{Restauration individuelle et réaménagement des espaces publics}

8 Ces dernières années, le milieu rural a connu de profondes mutations. Ce phénomène est d'ailleurs à rapprocher de l'évolution que la société française a connue dans son ensemble. Il trouve son origine à partir du XIX siècle avec les premiers départs de population vers les villes, et atteint son maximum au cours des années soixante.

9 A cette époque, le domaine rural qui reste fortement marqué par l'activité agricole, est en crise. Les villages ont perdu de nombreux habitants. Avec l'amélioration des moyens techniques et en particulier l'adoption du matériel lourd et son corollaire, le remembrement des terres cultivées, la Politique Agricole Commune (PAC) s'impose et cadre les nouvelles orientations de l'agriculture. Cette dernière, qui jusqu'ici avait servi de base à l'organisation des villages, perd désormais ce rôle. 
10 Alors que s'impose une économie très productive réformée dans ses choix, ses stratégies, ses échelles inédites, on assiste à l'arrivée de nouveaux habitants qui deviennent essentiels dans l'évolution du bâti rural.

11 L'espace rural en a fini de se confondre avec le territoire agricole. Ce phénomène qui trouve son origine dans la longue histoire de l'exode des campagnes, s'accentue quand le tarissement des départs concorde avec l'arrivée des nouveaux ruraux. Ce changement majeur esquissé dans les années soixante-dix est qualifié de renaissance rurale par les témoins les plus avertis qui ont noté cette inversion (B. Kayser, 1990). Alors que l'agriculture accumule les crises, entendons par là les suites de changements décisifs qui conduisent à créer de vastes exploitations poussées de l'avant par une logique capitaliste, les villages changent de contenu socio-démographique. L'activité agricole ne régit plus l'organisation du bâti et secondairement du réseau viaire. Les lois prônant la qualité de l'environnement, l'utilisation économe des richesses renouvelables et l'encouragement à la mise en place de projets durables plaident en faveur d'une séparation de l'agricole et du rural alors que les deux domaines s'étaient soutenus, confondus depuis toujours. Alors que s'affirme une économie agricole réformée par ses choix et ses finalités, bouleversée par des stratégies mobiles, perturbée par la prise en compte d'échelles de réflexions inédites, les ruraux investissent le village, y deviennent majoritaires et exercent un rôle essentiel dans l'évolution du bâti.

12 Dans le cadre du village renouvelé, le diagnostic de territoire s'articule autour de trois échelles pour analyser les modifications opérées. Les transformations concernent tout d'abord la parcelle individuelle où est située la maison, ses annexes et son environnement immédiat : le verger, le jardin et l'espace situé en avant de la maison. Cette première approche s'inscrit ensuite dans une analyse des linéaires. Il s'agit des espaces les plus visibles du village: la traversée par la route principale et les axes secondaires, les articulations entre le réseau viaire, les abords de bâtiments publics (mairie, école, église, lavoirs) et enfin les entrées de villages.

13 L'exposé de la trame de vie conduit en dernier lieu à énoncer les changements perceptibles à l'échelle globale du village, en particulier à partir de 1982, date de la promulgation des lois Deferre donnant aux communes la responsabilité et la gestion de leur territoire.

\section{Désir de campagne perçu et adaptation du cadre de vie résidentiel}

14 L'arrivée de citadins au sein des villages provoque de nombreux bouleversements qui affectent dans un premier temps le bâti.

Les besoins de confort, inhérents à l'époque, conduisent les propriétaires de fermes anciennes, qu'ils aient acquis leur bien par achat ou par héritage, à redistribuer les pièces à l'intérieur des bâtiments. Des sanitaires et des salles de bains sont aménagés en priorité, comme montre la figure 1. 
Figure 1 : Transformation d'une ferme de Bult (Vosges), construite en 1753
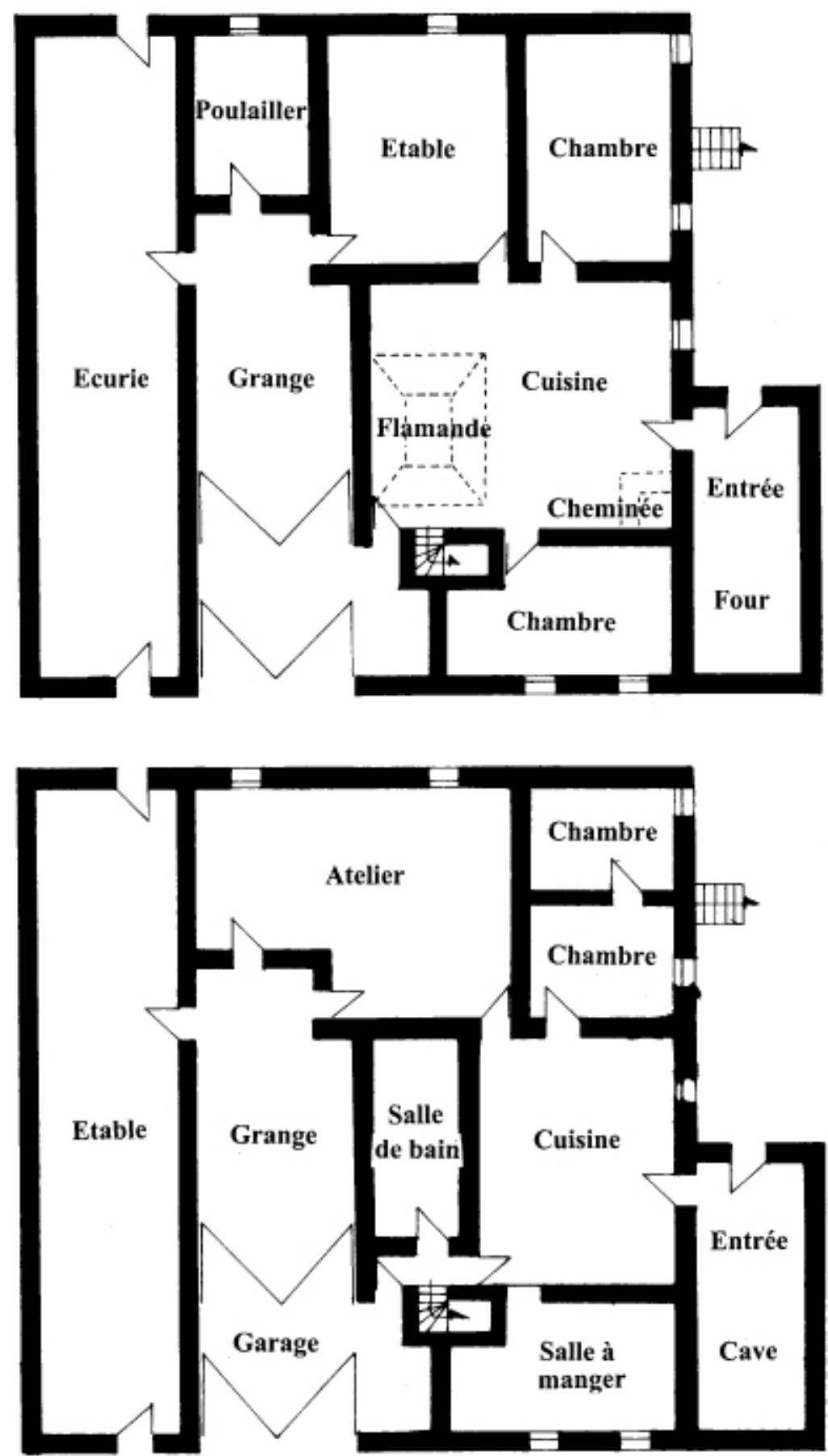

d'après Sayer C., sans date

16 Les espaces de vie ont été modifiés : une chambre est devenue une salle à manger, une autre a été recoupée au profit de deux pièces; la cuisine, dont les caractéristiques principales des fermes vosgiennes: flamande, cheminée à l'âtre, ont disparu, a été recoupée pour faire place à une salle de bain ; les fonctions agricoles ont disparu, avec la suppression du poulailler, la transformation du charri en garage. 
Ces bouleversements se répercutent en façade (figure 2). La composition originelle, avec des travées bien identifiables (celle de la partie habitation, de la grange et de l'étable) est remise en cause par l'intervention sur les percements. Les portes de grange devenues inutiles sont le plus souvent transformées. Les fenêtres sont agrandies, redistribuées. A l'extérieur, l'enduit utilisé est de moindre qualité. Les espaces extérieurs sont retravaillés.

Figure 2 : Transformation des façades (Autreville, Vosges)

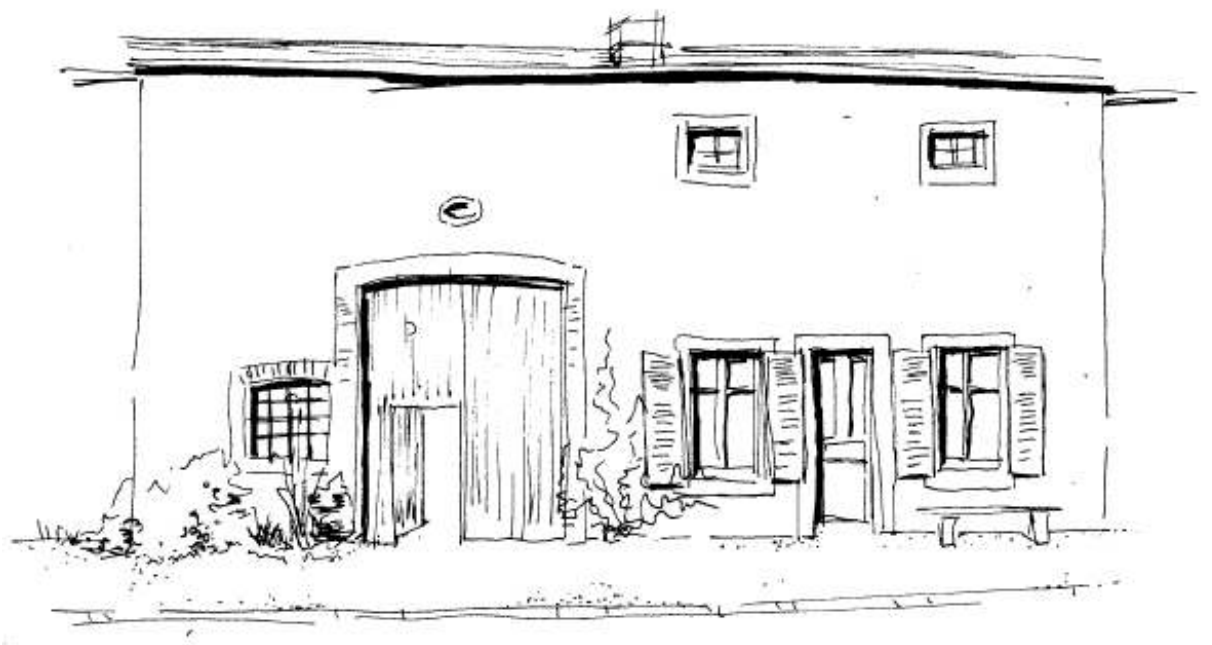

Façade traditionnelle

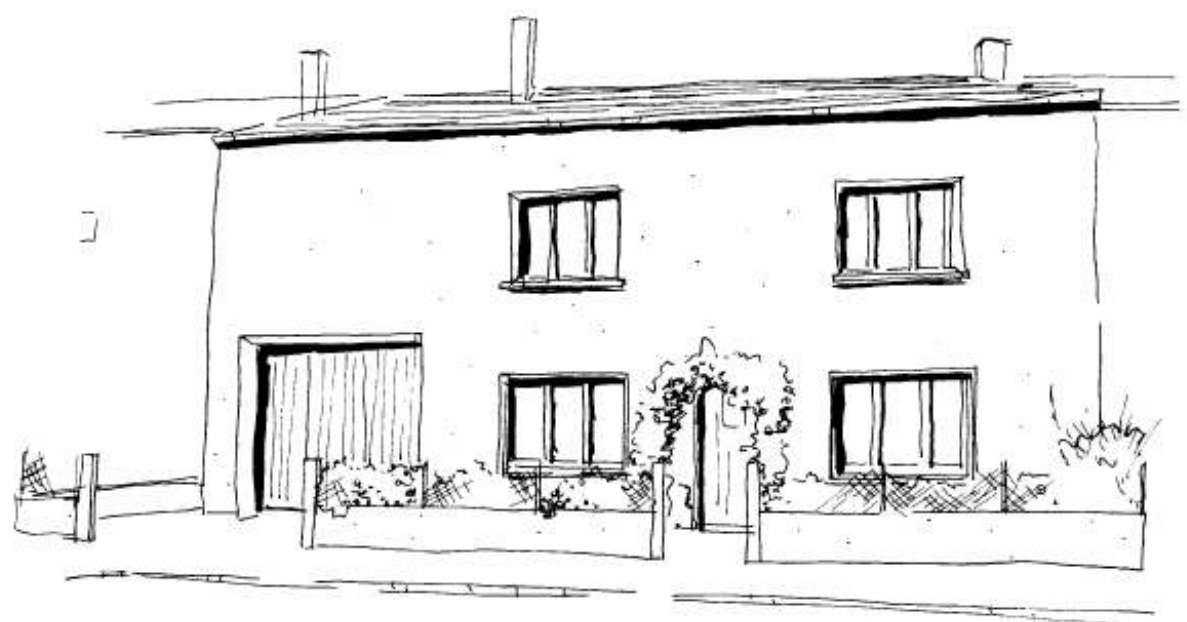

Façade très modifiée

Cette figure 2 représente deux façades de maisons à Autreville, situées au 11 et 17 rue de Nancy. La première montre l'image d'une ferme qui a conservé sa composition et son accompagnement végétal d'origine. Sur la seconde, l'image de la ferme traditionnelle a disparu, gommée par la modification de ses percements et de ses abords. Une porte de garage remplace la porte de grange, les fenêtres n'ont plus les mêmes proportions. L'usoir est clôturé par un muret et du grillage.

18 L'image du pavillon neuf est souvent appliquée comme modèle à la ferme dont les propriétaires ne sont plus agriculteurs. Tout signe distinctif de l'ancienne exploitation 
agricole est gommé volontairement. Si l'intérieur subit de nombreuses transformations, c'est la façade sur rue qui témoigne le plus des bouleversements.

Trop souvent, les percements sont modifiés. Les fenêtres d'origine accusées d'amener trop peu de lumière sont agrandies, élargies, altérant ainsi la composition originelle de la façade. Les menuiseries et volets en bois sont remplacés par du PVC; le double vitrage améliore le confort thermique de la maison, les volets roulants facilitent le réglage pour occulter la lumière.

La grange est transformée en garage. La porte trop grande est recoupée pour laisser la place à une porte de garage aux dimensions standards (2 㗐 $2,6 \mathrm{~m})$.

L'enduit traditionnel à la chaux en 3 couches (gobetis, couche d'accroche et couche de finition) est remplacé par un enduit monocouche de moindre résistance mécanique et qui donne priorité à des couleurs jusqu'ici inusitées, par exemple le blanc cassé. Les encadrements en pierre sont recouverts par une peinture. Dans certains cas, des vérandas sont ajoutées et servent de sas d'entrée ou de jardin d'hiver. Les tuiles canal disparaissent, remplacées par des tuiles mécaniques ou ciment.

L'usoir peut être clôturé par un grillage ou un muret avec une barrière parfois doublée d'une haie végétale (des thuyas le plus souvent).

Mais l'usoir, en devenant un jardin privatif, peut apparaitre comme une zone de contentieux avec la commune propriétaire concernée par l'entretien. Les arbustes, arbres d'ornement et fleurs domestiques remplacent désormais les arbres en espalier qui avaient l'avantage d'éponger l'humidité de l'assise du bâtiment.

24 A l'intérieur, c'est l'inconfort qui est combattu. Des salles de bains et des toilettes sont aménagées. L'ancienne cuisine souvent borgne est déplacée pour aller chercher la lumière du jour en façade, ou plus souvent sur l'arrière. Les pièces sont agrandies. Les granges ayant perdu leur fonction d'origine sont transformées en garages.

Certaines cloisons sont abattues, les pièces perdent leurs proportions d'origine Dans les aménagements à opérer, les habitants sont gênés par l'indice d'allongement des fermes. Ce dernier complique l'accès au jardin. Très souvent, l'espace de la grange est inutilisé, son grand volume ne pouvant être complètement occupé.

Le jardin à l'arrière est transformé en pelouse où trouvent place balançoires et tables de salons de jardin. Les arbres d'ornement cohabitent ou concurrencent les arbres fruitiers de l'ancien verger. Vu d'avion (A. Humbert, communication orale), le village lorrain semble retourné, la vie à la maison traduisant un regard beaucoup plus tourné vers le meix que vers l'usoir.

7 En conservant murs, charpentes et toitures, le propriétaire ambitionne avant tout de disposer d'un logement qui se rapproche de l'aspect offert par le pavillon. Ce changement d'affectation du bâti ne peut être qu'une suite de compromis pénalisants pour la structure originelle devenue peu lisible dans l'état actuel des réhabilitations.

Les traitements subis par le bâti et son environnement immédiat donnent un décor composite et contestable, dommageable pour les villages lorrains traditionnels. 


\section{Une rupture récente : la mise en valeur du village par et pour les néo-ruraux}

Parallèlement aux transformations des fermes des centres anciens, avec le développement des maisons individuelles, les villages sont également bouleversés dans leurs configurations d'origine (figure 3 ).

Figure 3 : Transformation des parcelles (Autreville)

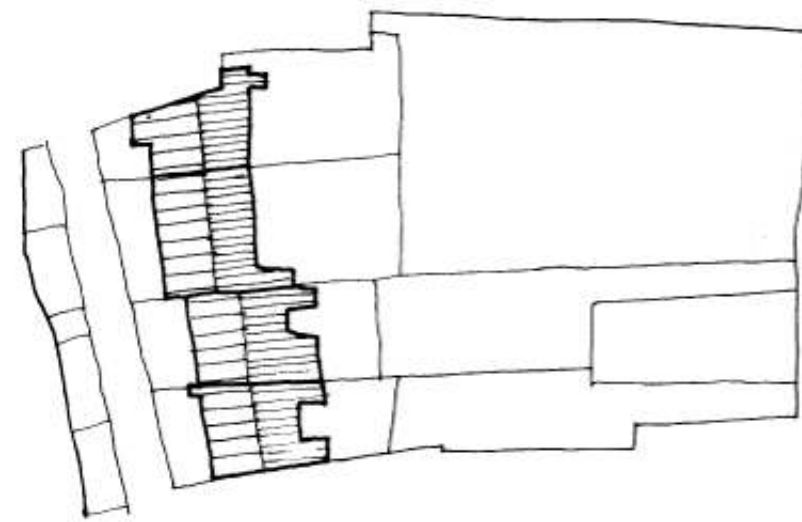

Usoirs Fermes Potagers Vergers

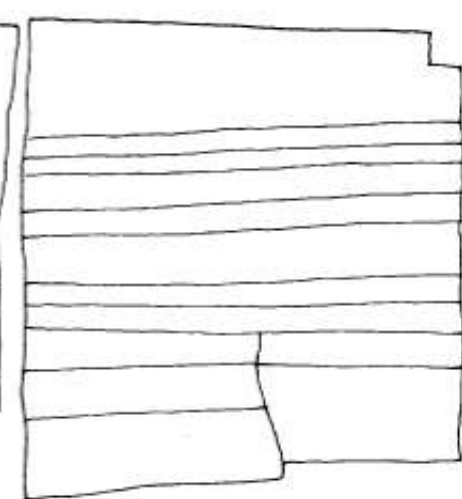

Chemin
Champs

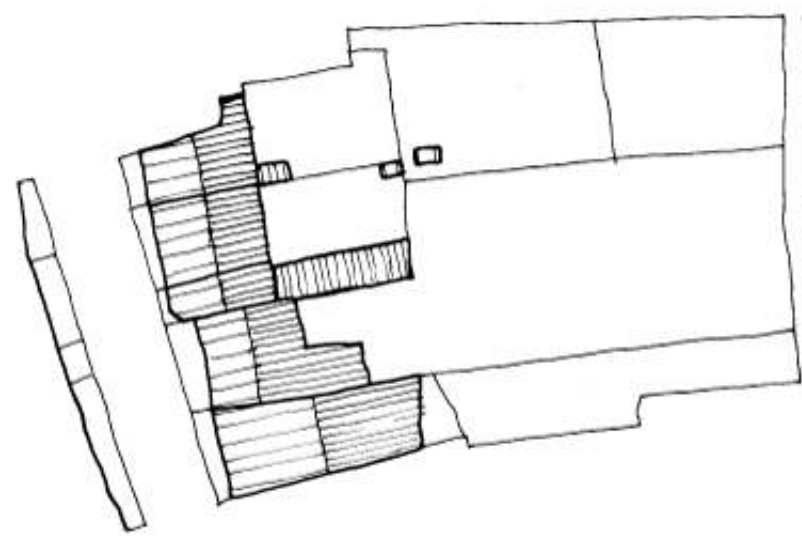

Chaussée Habitations
Jardins

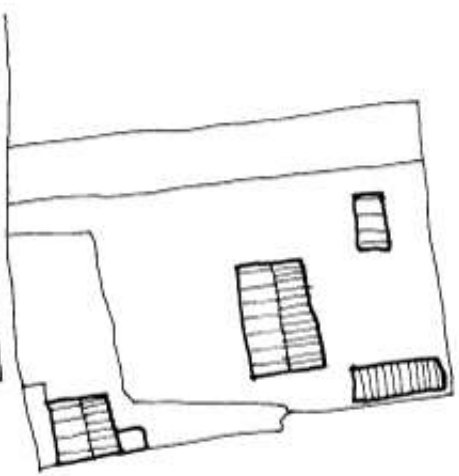

Chemin
Champs Habitations

Situation actuelle

La troisième transformation est celle du parcellaire qui a perdu ses fonctions agricoles d'origine au profit de fonctions résidentielles.

Le traitement des espaces de vie en commun fait l'objet d'interventions lourdes. Les entrées de village (figure 4), les traversées, les abords des édifices publics deviennent les enjeux majeurs des remodelages dans les petites communes. 


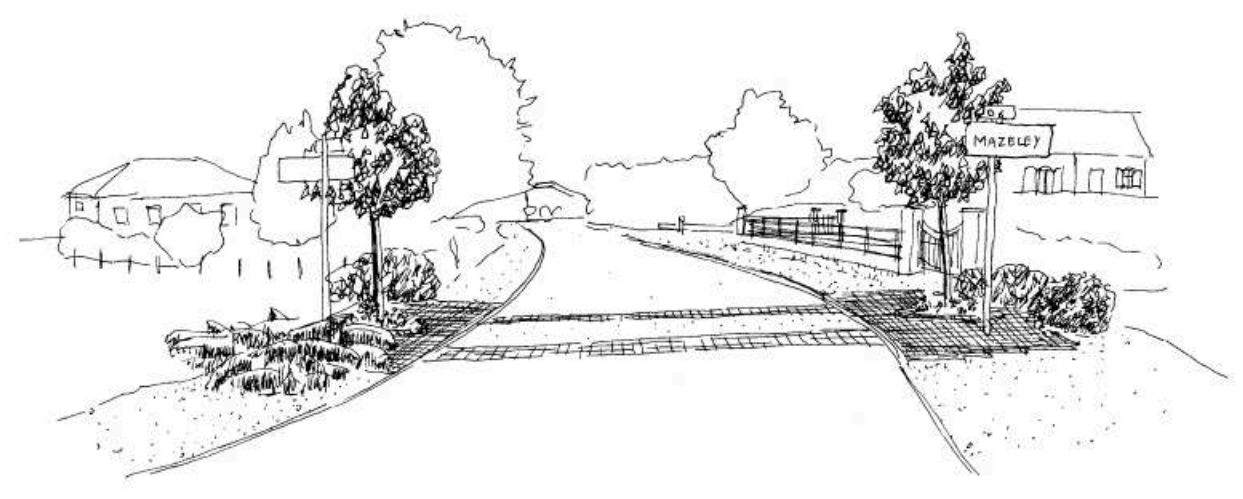

La figure montre un traitement contemporain de l'entrée de la commune vosgienne. Le projet a consisté à marquer les entrées du village par l'apport de végétation et de matériaux en contraste avec les éléments locaux: des arbres de couleurs en contraste avec les teintes environnantes, des arbustes et des fleurs d'agrément au milieu de champs, des pavés béton autobloquants traversant la chaussée. Ce travail s'apparente à un placage d'éléments.

d'après Huguenin, 2001

L'apparition du mot espace public traduit bien une nouvelle prise de conscience. Les lieux de vie collectifs représentent de nouveaux enjeux, définis dans un premier temps par rapport à une logique de ville, puis dans un second temps de village.

Associés aux notions d'aménagement et de milieu rural, les espaces publics sont développés, inscrits dans un contexte global qui leur est favorable. Ils concernent le cadre fonctionnel, social et culturel. Ils sont des lieux de rencontre où la vie sociale au cœur des villages a parfois du mal à reprendre. Avec la maison individuelle s'est développé le sentiment d'indépendance.

La rupture entamée avec la télévision, génératrice d'individualisme, s'aggrave avec la diffusion d'internet. Cela contribue à appauvrir l'idée de communauté soutenue par des politiques conviviales, relayant les participations passées à la ferveur religieuse.

Au cours des 25 dernières années, alors que chacun s'intéresse surtout à sa parcelle, des efforts sont consentis grâce à une volonté commune de revalorisation des espaces ouverts à tous.

Comme sur le bâti, l'intervention est issue directement du contexte urbain appliqué en milieu rural.

Ainsi sont retravaillés les lieux de vie collectifs en mettant de côté la sensibilité rurale. Formes, couleurs, mobiliers (traitement des sols, passages zébrés, lampadaires, etc.) sont introduits dans un contexte où la qualité des lieux est issue d'un équilibre précaire entre tous ces éléments.

La fin du village était jadis matérialisée par des croix et des alignements d'arbres. Déprise, rétraction du village ou à l'inverse croissance en continuité par rapport au bâti font que ces ancrages répondent assez mal à la réalité actuelle des limites. Aujourd'hui, les communes cherchent à justifier une nouvelle légitimité pour leur territoire en soignant les entrées les plus pratiquées par une triple opération : la mise en place de signalétiques, leur accompagnement par du fleurissement et enfin l'imposition de contraintes pour réguler et limiter les flux et la vitesse. Ces interventions globalement 
stéréotypées laissent assez peu de place à l'inventivité. Elles sont certes indispensables, mais posent des questions sur leur intégration avec le village.

Les édiles soucieux de mettre en avant le principe de précaution travaillent à améliorer la traversée des villages, et s'inscrivent à l'opposé d'une vieille tradition d'alignement qui n'est plus pratiquée. L'objectif actuel des mairies est de réduire la vitesse sur la chaussée, de mettre en valeur les édifices publics ou institutionnels (figure 5) ainsi que le patrimoine lié à l'eau. Ce travail s'accompagne d'une réflexion sur l'éclairage public, l'enterrement éventuel des réseaux aériens, le traitement du cheminement piétonnier, le stationnement des véhicules.

Figure 5 : Mazeley (Vosges) : réaménagement de l'îlot central

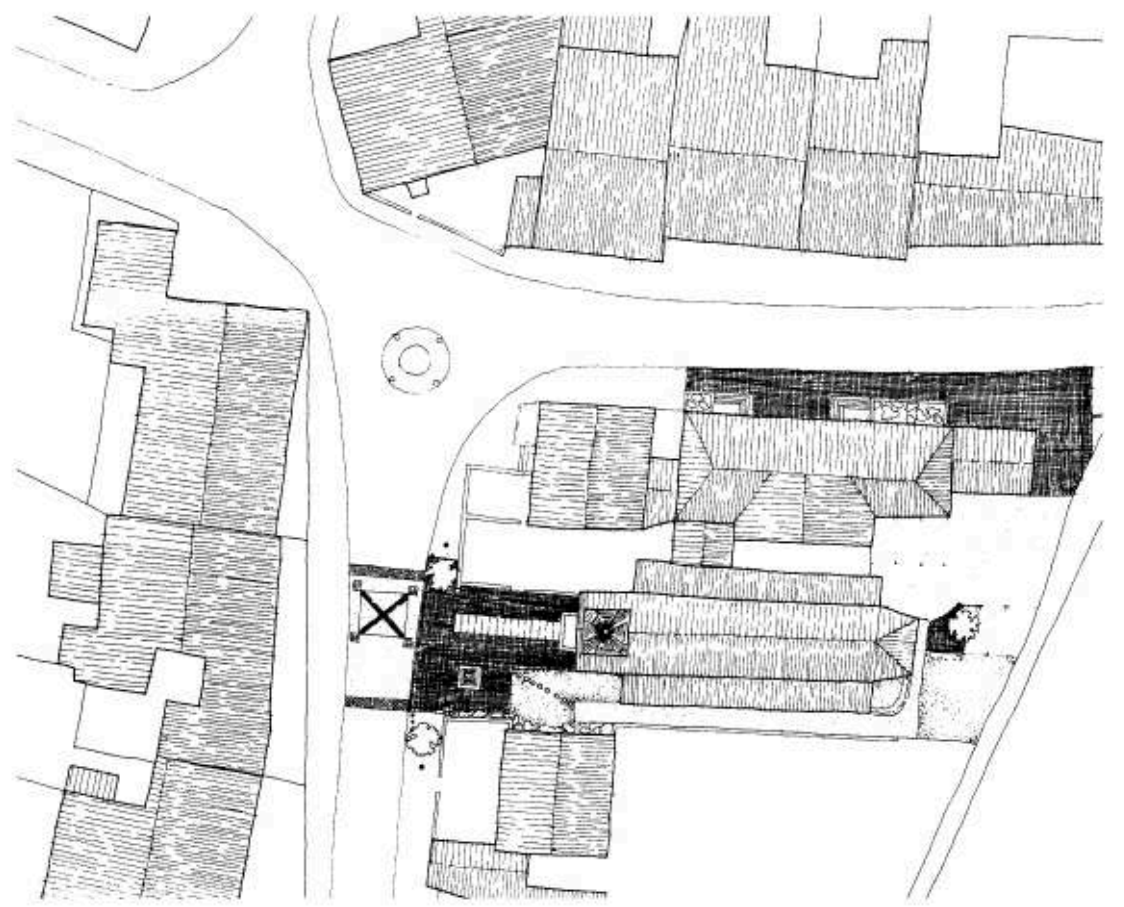

5a : Plan de l'îlot central 


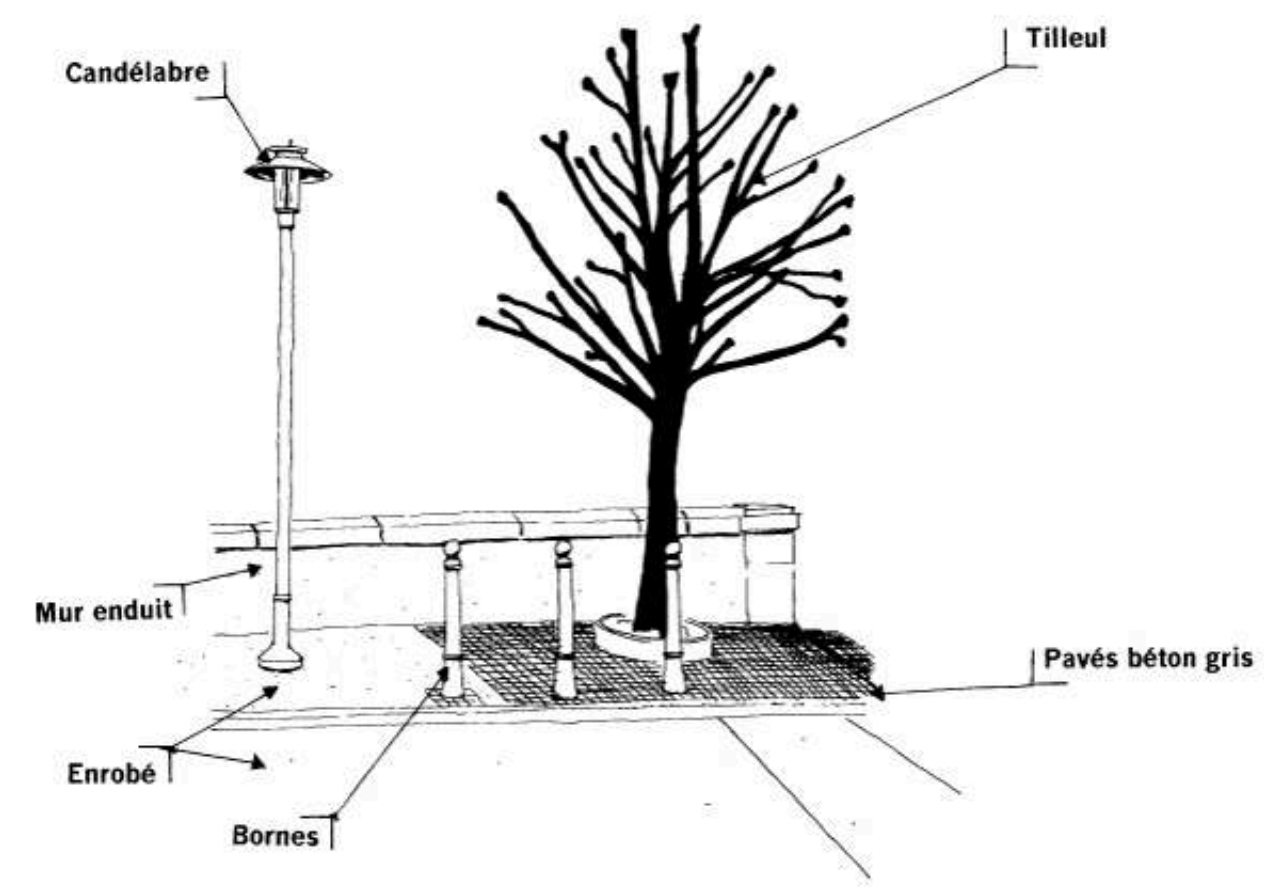

5b : Mazeley, le parvis

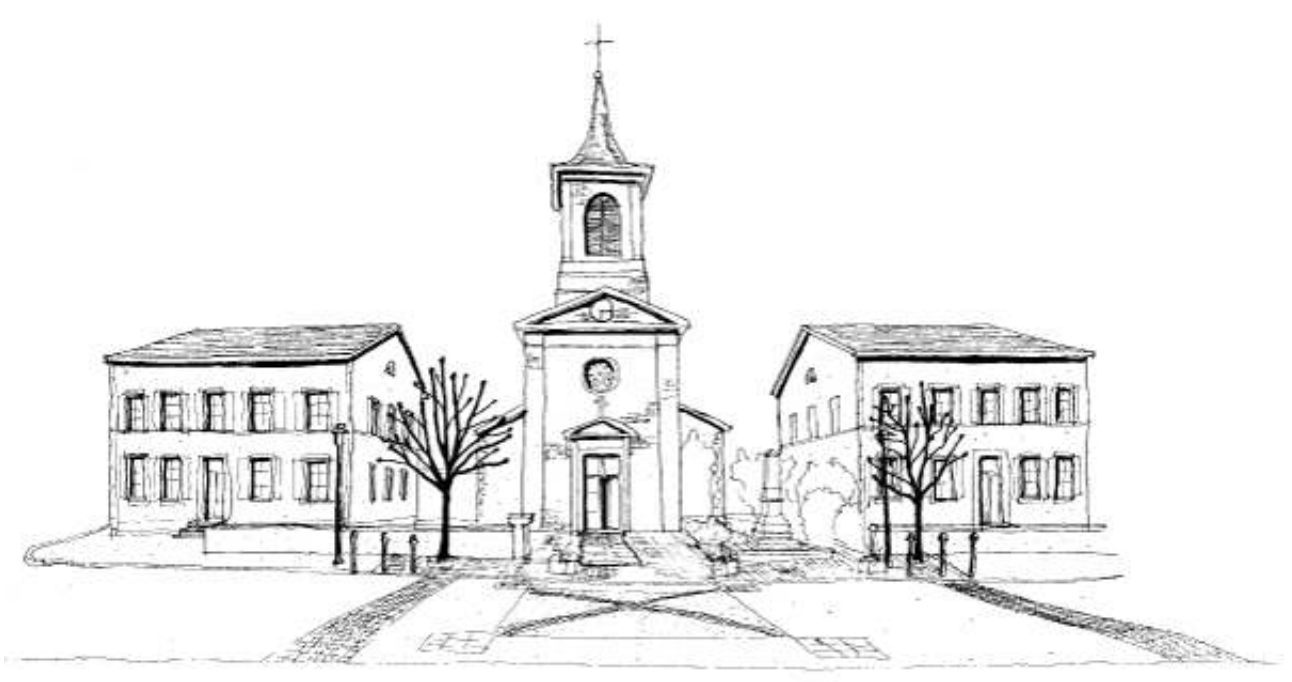

5c : Mazeley, détail du mobilier urbain

Les néo-ruraux affichent des comportements qui peuvent créer des paradoxes. D'un côté ils sont venus à la campagne pour profiter pleinement d'un mode de vie individualiste, d'autre part, ils souhaitent renouer avec des formes de convivialité conçues dans l'esprit de continuité des pratiques d'entraide jadis exprimées dans le calendrier agricole imposé par le ban et de formes de sociabilité (veillée, "couarail ", etc.). Tout cela était alors en cohésion avec la logique du bâti et les anciennes fonctionnalités de l'usoir. Idéalisées, parfois méconnues, les formes de cohérences sociales passées sont désormais adoptées par une population nouvelle et conduisent à s'interroger sur les aménagements à réaliser pour concrétiser des lieux d'ancrage, de rencontre des gens et pérenniser l'identité du village. 

s'annuler, placées entre les besoins nés des fonctions agricoles initiales et l'attente de nouvelles images favorisant l'esthétique.

\section{De nouvelles formes et repères pour les villages} par sa cathédrale. Au XIX ${ }^{e}$ siècle, l'héritage républicain puis les lois scolaires de J. Ferry font naître de nouveaux lieux de convergence. Il s'agit de l'école, qui incarne la garantie d'ascension sociale possible, de la mairie et du petit patrimoine lié à l'eau (lavoirs, égayoirs, fontaines). Ce double héritage est aujourd'hui malmené, parfois en voie de perte de ses fonctions par suite de la baisse de la pratique religieuse, des regroupements scolaires opérés, de l'arrivée du confort dans les maisons. Face à l'effacement des repères anciens, à relier au contexte d'effondrement des distancestemps, force est de s'interroger sur le nouveau sens de vie à donner au village si la communauté qui y demeure veut que l'habitat échappe au seul statut de site dortoir où chacun dispose de sa parcelle.

La figure 6 présente la situation d'un village fortement renouvelé, situé à $15 \mathrm{~km}$ d'Épinal et $12 \mathrm{~km}$ de Rambervillers. En une génération, Padoux a fortement étoffé son parc immobilier. De nouvelles maisons ont été bâties dans des parcelles laissées vacantes au centre du village et surtout en entrée de commune, au sud, sur le chemin de Sercœur. Ce triple processus d'étoffement se répète fréquemment, et se vérifie en particulier sur les marges des actuels SCOT en cours de délimitation. Les changements majeurs enregistrés ont souvent été opérés dans la précipitation, dans l'application de logiques individuelles facilitées par l'absence de cadrage (cartes communales actuelles, MARNU, qui doivent relayer les PLU dans les petites communes). Rétroactivement par rapport aux changements effectués, élus et acteurs de l'aménagement se concertent

Revue Géographique de l'Est, vol. 42 / 3 | 2002 
aujourd'hui avec les citoyens pour tenter de redonner du sens et de la cohésion au village. Ils aboutissent trop fréquemment à s'intéresser aux seuls espaces visibles pratiqués, traversés. Aussi, on aboutit plus au placage, au rajout de nouveaux éléments de décor qu'à une véritable politique de redéfinition de l'espace. Les actuelles transformations de l'agriculture, les transferts imposés des ateliers agricoles (installations classées) sont, pour les villages assez peu agrandis, une opportunité de récupérer à court terme du foncier et d'envisager une refonte en profondeur de leur territoire. Il y a là une chance à saisir pour concevoir, en anticipant sur l'avenir, des formes de croissance plus réfléchies et des modèles plus durables que celles et ceux qui ont été adoptés dans un passé proche.

Figure 6 : Padoux, un village « urbanisé » proche d'Épinal

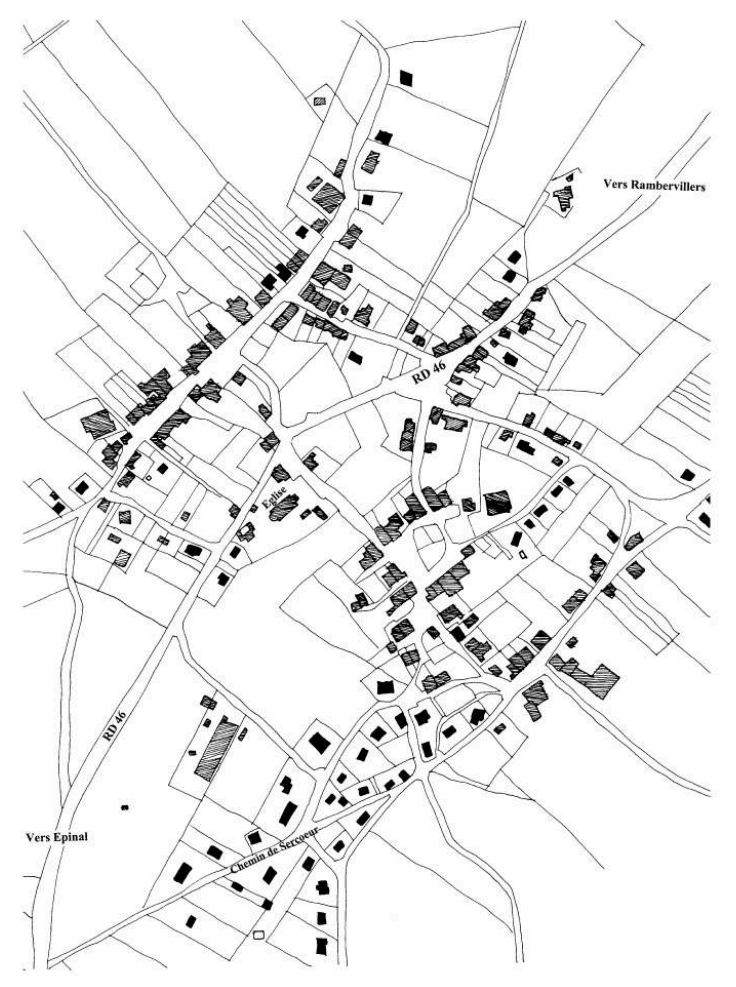

\section{Les scénarios d'évolution des bâtiments liés à l'agriculture}

\section{L'impossible pari de moderniser le bâti du village groupé}

Avec ses maisons alignées, profondes, jointives, formées d'un nombre de rains ou travées qui traduisait l'importance de l'exploitation (laboureur, semi-laboureur, pauvres), le village lorrain est resté fonctionnel tant qu'il a servi de cadre à une activité polyculturale céréalière abritée dans des fermes travaillant de cinq à trente hectares. L'agriculture était le levain de cohérence du village et l'organisation du bâti relevait d'une longue maturation d'un système agraire appuyé sur des pratiques collectives. Le respect du calendrier imposé par le ban traduisait un sens aigu de la cohésion sociale du groupe mais freinait toute émulation technologique. En l'espace d'un demi-siècle, la 
situation s'est totalement transformée et presque tous les archaïsmes agraires que l'on pouvait encore étudier vers 1960 ont disparu. Si l'activité agricole continue à assurer la gestion du territoire, elle est devenue secondaire, voire très minoritaire en nombre d'emplois, la plupart des villages n'ayant conservé que deux ou trois fermes assurées d'être transmissibles. La ferme du laboureur ou du «coq de village» occupait une position centrale qui était aussi celle de la reconnaissance sociale. Les fermes actuelles se sont dans un premier temps déplacées du cœur vers la périphérie du village. Aujourd'hui, elles quittent le village pour rester fonctionnelles, s'agrandir dans le respect de l'application des lois environnementales, plus particulièrement de celles qui concernent les rejets d'eaux usées (installations classées).

L'exploitation agricole est devenue une entreprise capitaliste. Elle est dopée par les restructurations foncières effectuées (le remembrement). L'exploitation agricole est poussée de l'avant par les économies d'échelles imposées. Ces dernières amènent à abandonner les solutions de compromis qui ont pu être retenues pendant la période de déperdition démographique précédant les actuelles formes de renaissance rurale perceptibles dès le recensement de 1975 (Arnould 1989, Farinelli 2001) et confirmées par la suite. L'exploitation ne peut plus fonctionner de façon éclatée en réutilisant des bâtis inoccupés. Sa productivité, son efficacité dépendent de l'optimum technique à attendre des installations, des capacités de stockage prévues, des applications légales à respecter, en particulier celles qui s'inscrivent dans les normes de la nouvelle loi sur l'eau en cours de négociation, enfin de l'insertion paysagère de l'entreprise agricole (Périgord, 2000). Les plans de développement durable (PDD) (Croix, 2000) ont été les initiateurs d'expériences préconisant des prises en compte globales des scénarios d'évolution des exploitations. L'actuelle mise en place des CTE (Contrats Territoriaux d'Exploitation) (Domas, 2000) poursuit cette ambition, change ou du moins infléchit la donne agricole placée entre systèmes très productif et post-productiviste.

Les exploitations agricoles maintenues au centre du village apparaissent aujourd'hui étranglées et ne répondent plus aux fonctionnalités attendues pour pérenniser l'exploitation et en faire un modèle reproductible, reprenable, adaptable par rapport à la mise en place de la récente loi SRU. L'application à venir de cette dernière est conçue pour être une avancée en matière d'urbanisme rural, un cadre rénovateur des politiques d'aménagement de l'espace. Elle devrait favoriser les projets de cohérence et non l'inverse trop souvent vérifié, autrement dit le mitage et la dilution de l'habitat.

Dans un passé proche, la mise en place autour du village d'un chemin périphérique a pu compléter l'opération de remembrement (par exemple à Omeray en Moselle). Cette réalisation a plutôt accompagné qu'anticipé l'évolution du positionnement des locaux agricoles. Elle permet aux exploitations situées en périphérie de faire transiter récoltes, bétail et matériaux encombrants par une seconde entrée.

51 L'actuelle restructuration des villages va dans un sens différents de ces retouches. Elle est surtout l'affaire des ruraux et néo-ruraux. On est là bien loin des cas de figure des villages reconstruits après les deux guerres (Gérard, 1995, 1996, 1997), en particulier après 1945. Ainsi, dans les Vosges, sur le contact du grès, Jeanménil s'était redressé en aérant le bâti, en abandonnant la jointivité pour des plans à cour ouverte ou en forme de L. Les bâtiments qui correspondaient à des trains de culture inscrits dans la fourchette $20-40$ ha étaient demeurés dans le village. 


\section{2. Étapes d'adaptation et scénarios de modernisation : des choix pour préserver la transmissibilité des exploitations}

\section{exclusive se mesurait à l'aune de la seule croissance de la production. Depuis 1992, les} inflexions menées en faveur d'une agriculture durable, plus économe que précédemment de moyens, s'inscrit dans une pause dans l'usage des intrants, dans une réflexion sur la désintensification. Cette période conduit ainsi à la rupture entre l'exploitation et le village. Les nouveaux bâtiments soumis à l'application des règles imposées aux installations classées sont au moins séparés de $100 \mathrm{~m}$ du village. Ils sont prioritairement conçus, positionnés, orientés pour optimiser la productivité d'un travail agricole très taylorisé puis informatisé. Dans leur conception est prise en compte l'efficacité et la surveillance d'un patrimoine technique considérable lié à une activité agricole devenue capitalistique (tableau 2).

Tableau 2 : Inscription du bâti agricole dans les différents cadrages de perception globale qui existent

\begin{tabular}{|l|l|l|l|l|}
\hline \multicolumn{1}{|c|}{ Exploitation } & \multicolumn{1}{|c|}{$\begin{array}{c}\text { Espace Institutionnel } \\
\text { (Communes, CDC) }\end{array}$} & $\begin{array}{c}\text { Echelle géographique } \\
\text { émergente (pays, bassin) }\end{array}$ \\
\hline $\begin{array}{l}\text { Agriculture capitalistique } \\
\text { infléchie par la durabilité }\end{array}$ & $\Rightarrow \quad \begin{array}{l}\text { Modèles globaux d'organisation } \\
\text { des territoires ruraux } \\
\text { Dont mise en application de la } \\
\text { SRU, de la loi sur l'eau, } \\
\text { des cohérences paysagères, } \\
\text { des CTE, etc. }\end{array}$ & $\Rightarrow$ & $\begin{array}{l}\text { Images de territoires } \\
\text { Projets de pays }\end{array}$ \\
\hline
\end{tabular}

Le bâti rural est aujourd'hui à l'épreuve de la mobilité (Mathieu et al., 2001), d'une pratique très élargie de l'espace vécu, sillonné en tous sens, consommant du territoire et à la recherche de formes d'assurance de convivialité et de sécurité. Le bâti agricole, soumis à des règles plus statiques que le précédent type de construction, est contraint de s'adapter à la nouvelle configuration de terres regroupées, obligé de structurer la vie de l'entreprise, d'anticiper les risques (séparer les fourrages des machines pour prévenir l'incendie).

Le tableau 3 résume les alternatives possibles et les choix retenus par les exploitants, sachant que chaque entreprise est un cas particulier défini par une mise en équation spécifique. 
Tableau 3 : Trois cas typiques de site et d'évolution d'exploitations agricoles lorraines, d'après $\mathrm{A}$. Dony, 2001 et enquête pour le CAUE 54

\begin{tabular}{|c|c|c|c|c|}
\hline Exploitation & Positionnement & $\begin{array}{l}\text { SAU } \\
\text { ha }\end{array}$ & $\begin{array}{l}\text { Vaches laitières/ } \\
\text { Total troupeau bovin }\end{array}$ & Stratégie possible \\
\hline A & Dans le village & 80 & $40 / 50$ & $\begin{array}{l}\text { A dispose d'une grosse exploitation déloca- } \\
\text { lisée située à } 30 \mathrm{~km} \text {. }\end{array}$ \\
\hline B & En périphérie & 90 & $50 / 104$ & $\begin{array}{l}\text { B est déclarée installation classée pour } \\
50 \text { vaches laitières et ne peut plus augmen- } \\
\text { ter ce type de cheptel. } \\
\text { B manque de place mais estime qu'à plus de } \\
50 \text { ans, il dispose de bonnes conditions de } \\
\text { travail, } 80 \text { ha étant situés à moins de } 1,5 \mathrm{~km} \\
\text { de la ferme, non transmissible en l'état. }\end{array}$ \\
\hline C & $\begin{array}{l}\text { A l'extérieur, } \\
\text { déplacée }\end{array}$ & 203 & $50 / 180$ & $\begin{array}{l}\text { normes, création d'un plan de masse fonc- } \\
\text { tionnel et distribution efficace des ateliers. C } \\
\text { continue à habiter au village, à } 200 \mathrm{~m} \text { de là. }\end{array}$ \\
\hline
\end{tabular}

\section{Conclusion}

L'analyse développée montre que les villages situés en secteur d'openfield font peau neuve. Les répercussions de la renaissance rurale affectent et transforment désormais de vastes territoires, même au-delà des zones des schémas de cohérence territoriale (SCOT).

Le bâti rural actuel évolue à plusieurs vitesses. Malgré les nombreuses formes de reprise (restauration des bâtis anciens, construction de pavillons, délocalisation des outils de la production agricole), il reste menacé dans ses structures cohérentes qui risquent de se déliter si des projets globaux d'harmonisation ne sont pas pensés à la double échelle des communes et des bassins de vie.

\section{BIBLIOGRAPHIE}

ARNoulD E. (1989). - La population des espaces de faible densité en Lorraine, étude démographique. Univ. Nancy 2, thèse de géographie, 584 p.

CROIX N. (dir.) (2000). - Des campagnes vivantes. Nantes, IGARUN, 696 p.

DOMAS A. (2000). - Les contrats territoriaux d'exploitation : contraintes juridiques et difficultés de mise en place. Paris, Courrier de l'Environnement de l'INRA,41, p. 81-86.

DoNY A. (2001). - Les bâtiments agricoles à travers évolution, aménagement, architecture, urbanisme rural et réglementaire. Univ. Nancy 2, mémoire de diplôme universitaire Aménagement et Urbanisme en milieu rural, 64 p.

FARINELLI X. (2001). - Le repeuplement des communes rurales, nécessité publique et désir individuel. Paris, Courrier de l'Environnement de l'INRA, 42, p. 15-27. 
GÉRARD Cl. (1990). — La maison rurale en Lorraine. Nonette, Créer, 151 p.

GÉRARD Cl. - Les villages de la reconstruction 1918-1928. Villages lorrains, Nancy, $\mathrm{n}^{\circ}$ 70, 1995, p. 9-13; n 72, 1996, p. 18-25; n 75, 1996, p. 15-22; n 78, 1997, p. 16-21.

HUGUENIN M. (2001). - L'aménagement d'espaces publics en milieu rural, les Vosges en exemple, Mémoire de l'école d'Architecture de Nancy, 4 dossiers.

KAYSER B. (1990). - La renaissance rurale. Sociologie des campagnes françaises. Paris, A. Colin.

KAYSER B. (1996). - Ils ont choisi la campagne. Paris, Aube, 205 p.

MATHIEU N., MOREL-Brochet A. (2001). - Essai sur l'habiter : le rural à l'épreuve de la mobilité in Berger A. (dir.) Dynamique rurale, environnement et stratégies spatiales. Montpellier, CNRS et Presses Universitaires, p. 459-466.

PeltRE J., GÉRARd C. (1978). - Les villages lorrains. Presses Universitaires, Nancy et éd. Serpenoise, Metz, $195 \mathrm{p}$.

PÉRIGORD M. (2000). - Politiques paysagères et dynamiques rurales, in Croix N. (dir.), Des campagnes vivantes, Rennes, Presses Universitaires, p. 97-108.

Planhol X. de (1969). - L'ancienne maison rurale en Lorraine. Norois, Poitiers, p. 315-336.

Reitel F. (1996). - A propos de l'openfield lorrain. Rev. Géo. de l'Est, 1-2, Nancy, p. 29-51.

SAYER C. - Essai de proposition méthodologique concernant trois communes vosgiennes : Nonzeville, Bult et Padoux. Univ. Nancy 2, mém. de maîtrise, géographie, sans date, 95 p.

\section{Sigles utilisés}

CDC : Communauté de communes.

CTE : Contrat territorial d'exploitation.

FGER : Fond de gestion de l'espace rural.

POS : Plan d'occupation des sols.

PLU : Plan local d'urbanisme (remplace le POS).

SCOT : Schéma de cohérence territoriale.

SRU : Loi de solidarité au renouvellement urbain.

\section{NOTES}

1. de Planhol, 1969 ; Peltre et Gérard, 1978 ; Gérard, 1990, 1995-1998 ; Reitel, 1996

\section{RÉSUMÉS}

Les villages d'openfield ont connu plusieurs changements majeurs articulés dans le temps: d'abord un long exode rural débuté après 1840 puis une renaissance rurale récente amorcée vers 
1970, accélérée depuis deux décennies. Ces transformations majeures modifient la cohérence du village aujourd'hui restauré, réorganisé, et mieux rénové (entrées de village, espaces collectifs, récréatifs, patrimoniaux). Elles s'inscrivent dans un contexte majoritairement post-agricole, dominé par des néo-ruraux qui transposent souvent la ville à la campagne.

Openfield Villages have experienced many major changes: first a long rural exodus beginning after 1840, then a recent rural renaissance beginning around 1970 and accelerating during the last two decades. These major transformations modified the coherence of the villages, today reorganised and renovated (village entrances, communal areas, recreation, cultural heritage).

Die Openfield-Dörfer haben mehrere grössere Veränderungen mitgemacht : zuerst eine ländliche Abwanderung, beginnend nach 1840, dann eine ländliche Renaissance, angefangen gegen 1970, beschleunigt seit zwei Jahrzehnten. Diese grösseren Transformationen modifizieren den inneren Zusammengang des Dorfes, heute reorganisiert und besser erneuert (Dorfeingänge, Gemeinschafts-und Erholungsflächen, Patrimonialland). Sie gehören zu einem überwiegend nachagrarischen Kontext, bestimmt durch eine neue Landbevölkerung, die oft die Stadt auf das Land verlagert.

\section{INDEX}

Schlüsselwörter : Lothringen, Openfield-Dorf, Renaissance

Keywords : Lorraine, openfied villages, renaissance

Mots-clés : Lorraine, renaissance, village d'openfield

\section{AUTEURS}

\section{MATHIEU HUGUENIN}

Architecte, Rectorat de l'Académie de Nancy-Metz - 2, rue Philippe de Gueldres CO n 13, 54035 Nancy Cedex

\section{JEAN-PIERRE HUSSON}

Professeur, département de géographie, Université Nancy 2 - BP 33-97, 54015 Nancy Cedex 